\title{
Monitoring the radio galaxy M87 with HAWC
}

\section{T. Capistrán, ${ }^{a, *}$ D. Avila Rojas, ${ }^{b}$ M.M. González, ${ }^{a}$ N. Fraija ${ }^{a}$ and R. Alfaro ${ }^{b}$ on behalf of the HAWC Collaboration}

(a complete list of authors can be found at the end of the proceedings)

${ }^{a}$ Instituto de Astronomía, Universidad Nacional Autónoma de México, Ciudad de Mexico, Mexico

${ }^{b}$ Instituto de Física, Universidad Nacional Autónoma de México, Ciudad de Mexico, Mexico

E-mail: tcapistran@astro.unam.mx, daniel_avila5@ciencias.unam.mx, magda@astro.unam.mx, nifraija@astro.unam.mx, ruben@fisica.unam.mx

Studies of radio galaxies at $\mathrm{TeV}$ energies are fascinating because their jets are misaligned concerning our sightline. Thus, it provides us with a unique opportunity to study the structure of their jets, the radiative processes, and the acceleration mechanisms involved in them. In addition, some radio galaxies have presented variability in their emission, like the giant radio galaxy M87, which has reported several activity periods. Due to its duty cycle $>95 \%$ and instantaneous field of view of $2 \mathrm{sr}$, HAWC provides daily monitoring of variable sources visible from the Northern Hemisphere. In this work, we show the results of monitoring M87 between January 2015 and December 2018. HAWC's observations are consistent with the low activity state reported by other instruments (like H.E.S.S and MAGIC). However, after September 2017 ( MJD 58000), the HAWC measurements of M87 show hints of higher activity.

$37^{\text {th }}$ International Cosmic Ray Conference (ICRC 2021)

July 12th-23rd, 2021

Online - Berlin, Germany

\footnotetext{
*Presenter
} 


\section{Introduction}

The majority of bright gamma-ray sources are active galactic nuclei (AGN) which have observed with bolometric luminosities up to $10^{48}$ to $10^{49} \mathrm{ergs} / \mathrm{s}$ [1]. Blazars, a subclass of AGN that launches a relativistic jet aligned with our line-of-sight [2], are known to show large variability in the whole electromagnetic spectrum. Blazars are commonly divided in BL Lac objects and Flat Spectrum Radio Quasars (FSRQ) [3]. Radio galaxies are a subclass of AGN that launch jets oriented at large inclination angles with respect to us $\left(\theta>10^{\circ}\right)$ [4]. These objects give an excellent and unique opportunity to study the AGNs from another perspective owing to the ability to observe all their structure (e.g., their nucleus, jets and giant lobes). Thus, these kinds of sources help us research the physical processes of gamma-ray production and the location of the emission region.

The radio galaxies are more difficult to detect in the gamma-ray band due to their misaligned jet, which makes the non-thermal radiation to be faint. Despite this, 30 of these sources have been detected above $100 \mathrm{MeV}$, and six of them at very-high energies (>100 GeV) [4]. Regardless of the low number of these sources detected in the TeV regimen, new studies of multi-wavelength observations have revealed a fast variability in the radio galaxies M87 and IC 310 (order of 4.8 $\min )[4-7]$.

In this work, we made a study on M87 using the High-Altitude Water Cherenkov (HAWC) gamma-ray Observatory. In Section 2 we present a summary of the events more representative in the TeV regime of M87. In Section 3 we describe the monitoring of M87 using the HAWC Observatory. Finally, we summarise in Section 4 the work and remark the importance of this kind of study.

\section{The Radio Galaxy M87}

M87 is a giant elliptical radio galaxy located in the Virgo Cluster and was the first radio galaxy detected in the $\mathrm{TeV}$ regimen by High-Energy-Gamma-Ray Astronomy (HEGRA) observatory in 1998 [8]. Like other $\mathrm{TeV}$ radio galaxies, M87 hosts a supermassive black hole $\left(6 \times 10^{9} M_{\odot}\right)$; it is the second nearest radio galaxy to the Earth $(\mathrm{z}=0.0044)$; it is classified as Fanaroff \& Riley class I according with morphology in radio; and its relativistic jet has an angle between $15^{\circ}$ and $25^{\circ}$ with respect to our line of sight [9].

Radio galaxies present variability on multiple time scales, similar to Blazars [10, 11]. This is the reason gamma-ray observatories started to monitor these kinds of sources. The results on M87 campaigns reports three flaring states during the years 2005, 2008 and 2010 [12]. After 2010, it has been in a quiescent or low-emission state. It was reported by MAGIC observatory and also observed from gamma-ray to radio band [9]. The flux normalization at $1 \mathrm{TeV}$ of this source in a quiescent state is between [2-7] $\times 10^{-13} \mathrm{~cm}^{-2} \mathrm{~s}^{-1} \mathrm{TeV}^{-1}$ reported by MAGIC, VERITAS and H.E.S.S. and during the flaring state is around $[1-5] \times 10^{-12} \mathrm{~cm}^{-2} \mathrm{~s}^{-1} \mathrm{TeV}^{-1}$. In both cases, the spectral model used is a simple power-law function with an index of $\sim 2.3$ with an exception for the observation of 2004 by H.E.S.S. and 2012 by VERITAS, where they used a spectral of $~ 2.6$. 


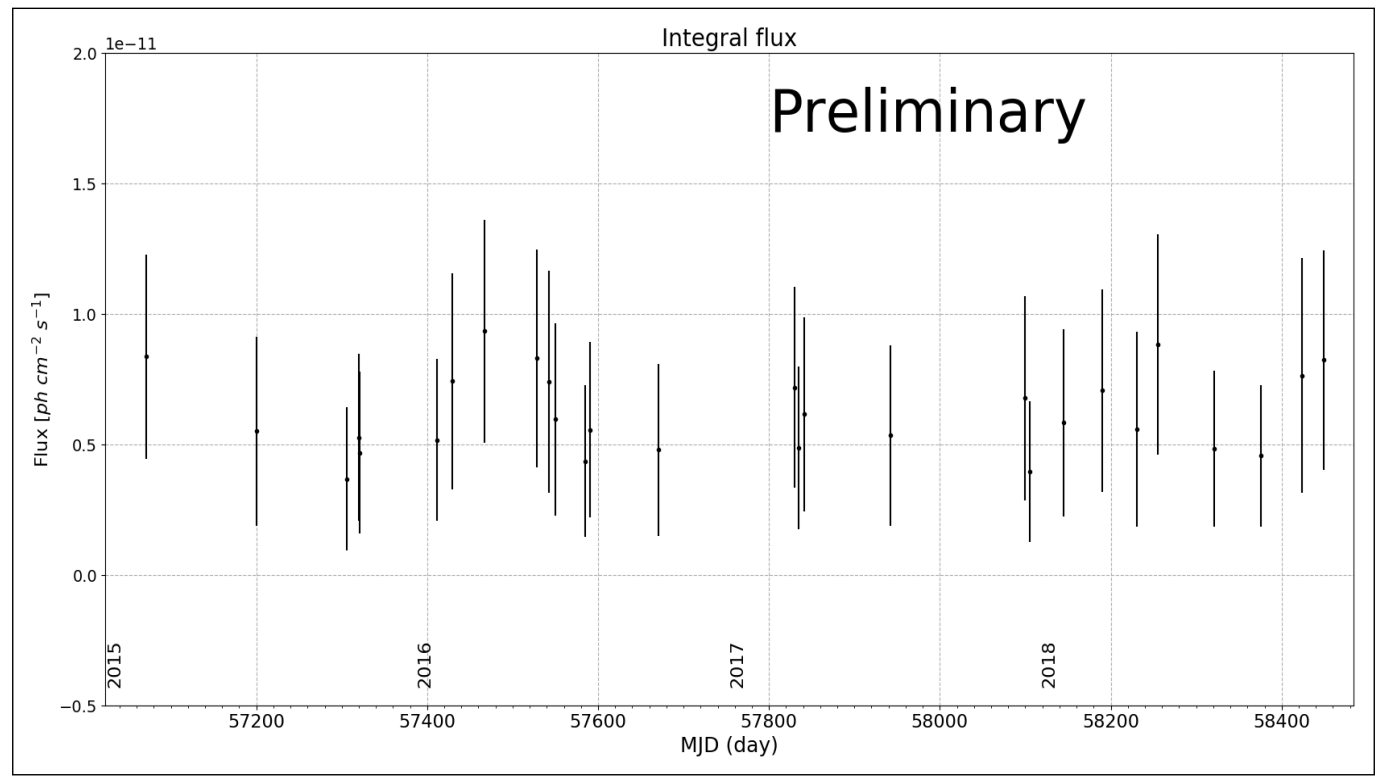

Figure 1: Daily light curve of M87 from January 1st 2015 (MJD 57023) to December 8th 2018 (MJD 58460). Here only are shown all days with a significance greater than 2 .

HAWC is an observatory that operates almost all day (duty cycle $>95 \%$ ), so it makes a perfect instrument to monitor the source every day. Another critical point of this experiment is the wide field of view that can detect sources with declination from $-26^{\circ}$ (a small part of the South sky) to $64^{\circ}$ (North sky), which M87 is inside of it. Another more is the detection of gamma-ray at TeV [13].

\section{HAWC results}

The analysis performed to obtain the light curve of M87 is the same as Abeysekara et. at [14] used for reporting the daily monitoring of two blazars and the Crab Nebula of the first 17 months of HAWC. This analysis is computed with the Likelihood fitting Framework (LIFF) package[15]. This package estimates the flux and significance using the maximum likelihood method to fit a physical spectral model convolved with the HAWC detector response to the data.

The daily light curve is obtained using HAWC data from January 1st 2015 to December 8th 2018. A simple power-law spectrum model is used, with a pivot energy of $1 \mathrm{TeV}$ and an index of 2.3. The Franceschini model for EBL attenuation is also used. Two cuts were applied on the light curve: first, a minimum exposure of 4.2 hours, and second, only plot days with a significance greater than $2 \sigma$. Figure 1 shows the daily light curve using the previous quality cut. During this period, the fluxes of M87 have a value between [0.3-1.0] $\times 10^{-11} \mathrm{ph} \mathrm{cm}^{-2} \mathrm{~s}^{-1}$.

Figure 2 show the monthly light curve. This curve uses the same period of HAWC data and the same spectral model than the daily light curve. The main difference in this plot is the calculation of the upper limits at the confidence level of $95 \%$. The integral flux point and the upper limit values are consistent with a non-active state. These results on M87 are consistent with the quiescent state observed in this radio galaxy. 


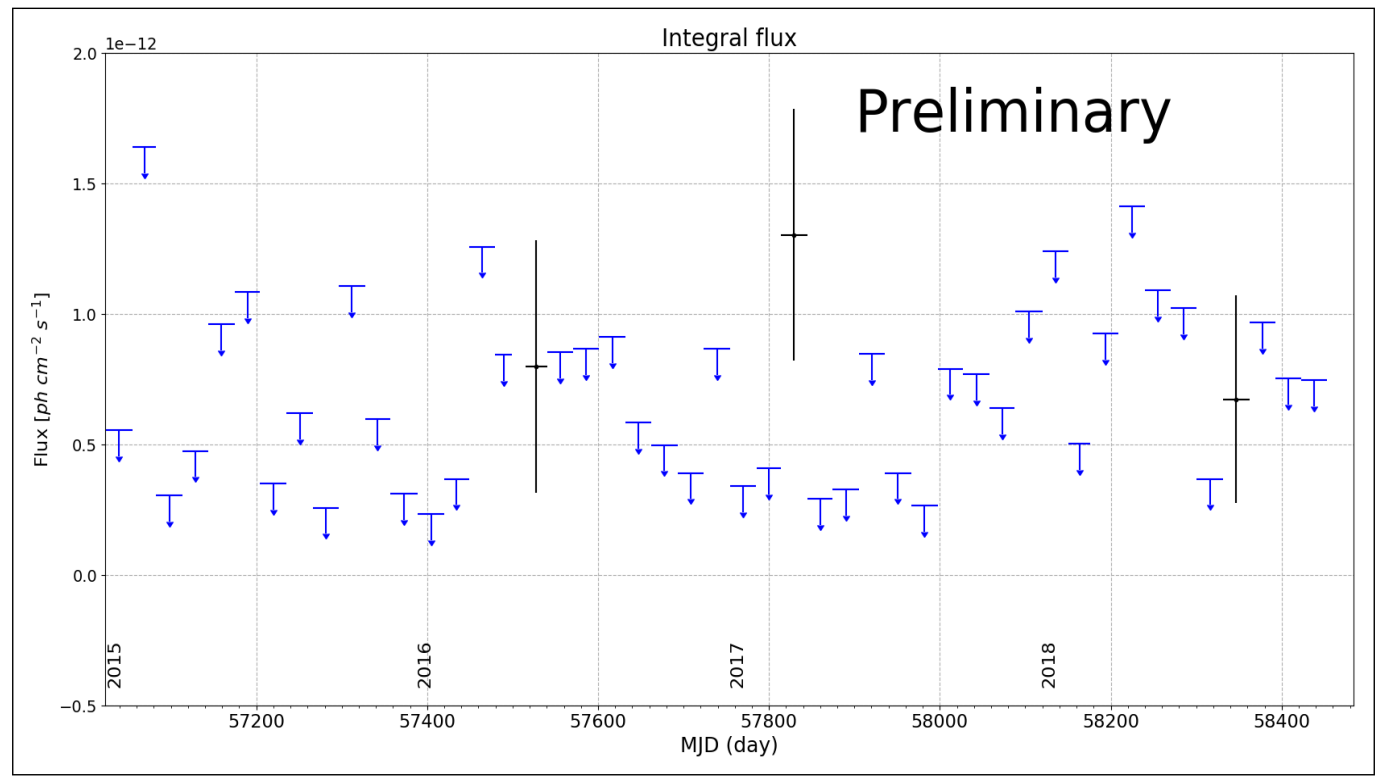

Figure 2: Monthly light curve of M87 from January 1st 2015 (MJD 57023) to December 8th 2018 (MJD 58560). The black marker are the months that has a significance greater than $2 \sigma$, the rest, blue ones, are the upper limit at $95 \%$.

We then looked at integrating the data around M87 over time. A cumulative significance was calculated adding a month by a month until the four years were covered and it is shown in Figure 3. We can see that after September 2017 (MJD 58000) the significance starts to rise steadily. Using the full livetime of the analysis, the final significance obtained is $1.8 \sigma$, while restricting the livetime by using data above MJD 58000, the significance increases up to $2.6 \sigma$.

\section{Conclusion}

M87 has been monitored using four years of HAWC data with two different time scales. During this period of observation, M87 was found to be in a nonactive state. However, by restricting the live-time using data after September 2017, we observe a hint of evidence of TeV emission from M87. It could mean that this radiogalaxy is becoming active again. HAWC will keep monitoring the evolution of this source.

In this work, the significance obtained in the radio galaxy M87 is around $2 \sigma$ using around four years of data. New improvements on the analysis are under way and some preliminary results show that this radio galaxy is now significant. We will present this in the future.

\section{Acknowledgments}

We acknowledge the support from: the US National Science Foundation (NSF); the US Department of Energy Office of High-Energy Physics; the Laboratory Directed Research and Development (LDRD) program of Los Alamos National Laboratory; Consejo Nacional de Ciencia y Tecnología (CONACyT), México, grants 271051, 232656, 260378, 179588, 254964, 258865, 243290, 132197, 


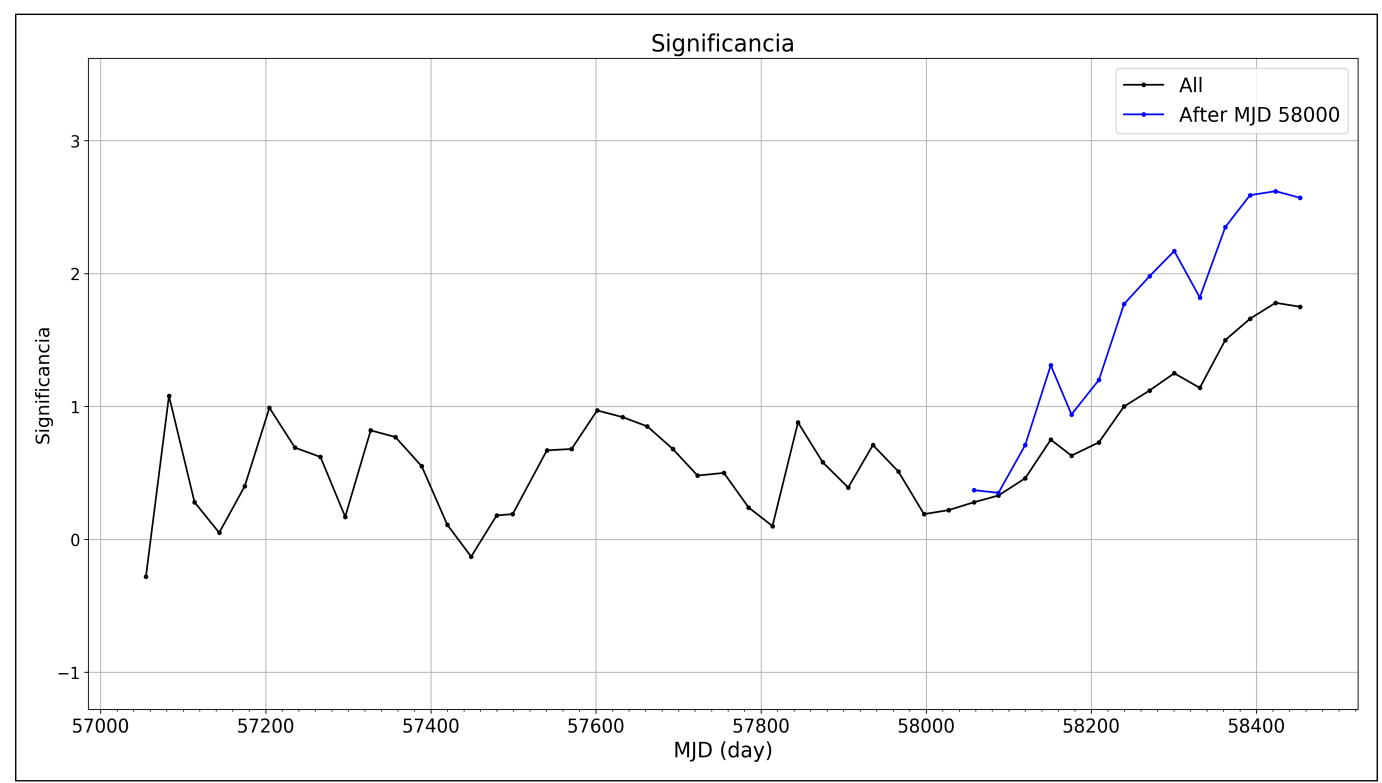

Figure 3: Cumulative significance is shown by adding month-by-month data from January 2015 (MJD 57023) to December 2018 (MJD 58560). After September 2017 (MJD 58000), the significance starts to increase.

A1-S-46288, A1-S-22784, cátedras 873, 1563, 341, 323, Red HAWC, México; DGAPA-UNAM grants IG101320, IN111716-3, IN111419, IA102019, IN110621, IN110521; VIEP-BUAP; PIFI 2012, 2013, PROFOCIE 2014, 2015; the University of Wisconsin Alumni Research Foundation; the Institute of Geophysics, Planetary Physics, and Signatures at Los Alamos National Laboratory; Polish Science Centre grant, DEC-2017/27/B/ST9/02272; Coordinación de la Investigación Científica de la Universidad Michoacana; Royal Society - Newton Advanced Fellowship 180385; Generalitat Valenciana, grant CIDEGENT/2018/034; Chulalongkorn University's CUniverse (CUAASC) grant; Coordinación General Académica e Innovación (CGAI-UdeG), PRODEP-SEP UDG-CA499; Institute of Cosmic Ray Research (ICRR), University of Tokyo, H.F. acknowledges support by NASA under award number 80GSFC21M0002. We also acknowledge the significant contributions over many years of Stefan Westerhoff, Gaurang Yodh and Arnulfo Zepeda Dominguez, all deceased members of the HAWC collaboration. Thanks to Scott Delay, Luciano Díaz and Eduardo Murrieta for technical support.

\section{References}

[1] A. Albert et al., A Survey of Active Galaxies at TeV Photon Energies with the HAWC Gamma-Ray Observatory, apj 907 (2021) 67 [2009. 09039].

[2] R.D. Blandford and A. Königl, Relativistic jets as compact radio sources, apj 232 (1979) 34.

[3] M.J.M. Marcha, I.W.A. Browne, C.D. Impey and P.S. Smith, Optical spectroscopy and polarization of a new sample of optically bright flat radio spectrum sources, mnras $\mathbf{2 8 1}$ (1996) 425. 
[4] FERMI-LAT collaboration, Gamma-ray emission in radio galaxies, from $\mathrm{MeV}$ to $\mathrm{TeV}$, in IAU Symposium 342: Perseus in Sicily: from black hole to cluster outskirts, 9, 2018, DOI [1809.08074].

[5] J. Aleksic et al., Black hole lightning due to particle acceleration at subhorizon scales, Science 346 (2014) 1080 [1412 . 4936].

[6] N. Fraija and A. Marinelli, Neutrino, $\gamma$-Ray, and Cosmic-Ray Fluxes from the Core of the Closest Radio Galaxies, apj 830 (2016) 81 [1607.04633].

[7] N. Fraija, A. Marinelli, A. Galván-Gámez and E. Aguilar-Ruiz, Modeling the spectral energy distribution of the radio galaxy IC310, Astroparticle Physics 89 (2017) 14 [1701.06173].

[8] F. Aharonian et al., Is the giant radio galaxy $M 87$ a TeV gamma-ray emitter?, aap 403 (2003) L1 [astro-ph/0302155].

[9] V.A. Acciari et al., Monitoring of the radio galaxy M 87 during a low-emission state from 2012 to 2015 with MAGIC, Monthly Notices of the Royal Astronomical Society 492 (2020) 5354.

[10] D. Glawion, Radio galaxies at tev energies: Preface, Galaxies 8 (2020).

[11] B. Rani, Radio Galaxies-The TeV Challenge, Galaxies 7 (2019) 23 [1811.00567].

[12] F.M. Rieger and A. Levinson, Radio Galaxies at VHE energies, Galaxies 6 (2018) 116 [1810.05409].

[13] A.U. Abeysekara et al., Observation of the Crab Nebula with the HAWC Gamma-Ray Observatory, apj 843 (2017) 39 [1701.01778].

[14] A.U. Abeysekara et al., Daily Monitoring of TeV Gamma-Ray Emission from Mrk 421, Mrk 501, and the Crab Nebula with HAWC, apj 841 (2017) 100 [1703.06968].

[15] P.W. Younk et al., A high-level analysis framework for HAWC, in 34th International Cosmic Ray Conference (ICRC2015), vol. 34 of International Cosmic Ray Conference, p. 948, July, 2015 [1508 . 07479]. 


\section{Full Authors List: HAWC Collaboration}

A.U. Abeysekara ${ }^{48}$, A. Albert ${ }^{21}$, R. Alfaro ${ }^{14}$, C. Alvarez ${ }^{41}$, J.D. Álvarez ${ }^{40}$, J.R. Angeles Camacho ${ }^{14}$, J.C. Arteaga-Velázquez ${ }^{40}$, K. P. Arunbabu $^{17}$, D. Avila Rojas ${ }^{14}$, H.A. Ayala Solares ${ }^{28}$, R. Babu ${ }^{25}$, V. Baghmanyan ${ }^{15}$, A.S. Barber ${ }^{48}$, J. Becerra Gonzalez ${ }^{11}$, E. BelmontMoreno $^{14}$, S.Y. BenZvi ${ }^{29}$, D. Berley ${ }^{39}$, C. Brisbois ${ }^{39}$, K.S. Caballero-Mora ${ }^{41}$, T. Capistrán ${ }^{12}$, A. Carramiñana ${ }^{18}$, S. Casanova ${ }^{15}$, O. Chaparro-Amaro $^{3}$, U. Cotti ${ }^{40}$, J. Cotzomi ${ }^{8}$, S. Coutiño de León ${ }^{18}$, E. De la Fuente ${ }^{46}$, C. de León ${ }^{40}$, L. Diaz-Cruz ${ }^{8}$, R. Diaz Hernandez ${ }^{18}$, J.C. Díaz-Vélez ${ }^{46}$, B.L. Dingus ${ }^{21}$, M. Durocher ${ }^{21}$, M.A. DuVernois ${ }^{45}$, R.W. Ellsworth ${ }^{39}$, K. Engel ${ }^{39}$, C. Espinoza ${ }^{14}$, K.L. Fan ${ }^{39}$, K. Fang $^{45}$, M. Fernández Alonso ${ }^{28}$, B. Fick ${ }^{25}$, H. Fleischhack ${ }^{51,11,52}$, J.L. Flores ${ }^{46}$, N.I. Fraija ${ }^{12}$, D. Garcia ${ }^{4}$, J.A. García-González ${ }^{20}$, J. L. García-Luna ${ }^{46}$, G. García-Torales ${ }^{46}$, F. Garfias ${ }^{12}$, G. Giacinti ${ }^{22}$, H. Goksu ${ }^{22}$, M.M. González ${ }^{12}$, J.A. Goodman ${ }^{39}$, J.P. Harding ${ }^{21}$, S. Hernandez ${ }^{14}$, I. Herzog ${ }^{25}$, J. Hinton ${ }^{22}$, B. Hona ${ }^{48}$, D. Huang ${ }^{25}$, F. Hueyotl-Zahuantitla ${ }^{41}$, C.M. Hui ${ }^{23}$, B. Humensky ${ }^{39}$, P. Hüntemeyer ${ }^{25}$, A. Iriarte ${ }^{12}$, A. Jardin-Blicq ${ }^{22,49,50}$, H. Jhee ${ }^{43}$, V. Joshi ${ }^{7}$, D. Kieda ${ }^{48}$, G J. Kunde ${ }^{21}$, S. Kunwar ${ }^{22}$, A. Lara ${ }^{17}$, J. Lee ${ }^{43}$, W.H. Lee ${ }^{12}$, D. Lennarz ${ }^{9}$, H. León Vargas ${ }^{14}$, J. Linnemann ${ }^{24}$, A.L. Longinotti ${ }^{12}$, R. López-Coto ${ }^{19}$, G. Luis-Raya ${ }^{44}$, J. Lundeen ${ }^{24}$, K. Malone ${ }^{21}$, V. Marandon $^{22}$, O. Martinez ${ }^{8}$, I. Martinez-Castellanos ${ }^{39}$, H. Martínez-Huerta ${ }^{38}$, J. Martínez-Castro ${ }^{3}$, J.A.J. Matthews ${ }^{42}$, J. McEnery ${ }^{11}$, P. Miranda-Romagnoli $^{34}$, J.A. Morales-Soto ${ }^{40}$, E. Moreno ${ }^{8}$, M. Mostafá ${ }^{28}$, A. Nayerhoda ${ }^{15}$, L. Nellen ${ }^{13}$, M. Newbold ${ }^{48}$, M.U. Nisa ${ }^{24}$, R. Noriega-Papaqui ${ }^{34}$, L. Olivera-Nieto ${ }^{22}$, N. Omodei ${ }^{32}$, A. Peisker ${ }^{24}$, Y. Pérez Araujo ${ }^{12}$, E.G. Pérez-Pérez ${ }^{44}$, C.D. Rho ${ }^{43}$, C. Rivière ${ }^{39}$, D. Rosa-Gonzalez ${ }^{18}$, E. Ruiz-Velasco ${ }^{22}$, J. Ryan ${ }^{26}$, H. Salazar ${ }^{8}$, F. Salesa Greus ${ }^{15,53}$, A. Sandoval ${ }^{14}$, M. Schneider ${ }^{39}$, H. Schoorlemmer ${ }^{22}$, J. Serna-Franco ${ }^{14}$, G. Sinnis ${ }^{21}$, A.J. Smith ${ }^{39}$, R.W. Springer ${ }^{48}$, P. Surajbali ${ }^{22}$, I. Taboada ${ }^{9}$, M. Tanner ${ }^{28}$, K. Tollefson ${ }^{24}$, I. Torres ${ }^{18}$, R. Torres-Escobedo $^{30}$, R. Turner ${ }^{25}$, F. Ureña-Mena ${ }^{18}$, L. Villaseñor ${ }^{8}$, X. Wang ${ }^{25}$, I.J. Watson ${ }^{43}$, T. Weisgarber ${ }^{45}$, F. Werner ${ }^{22}$, E. Willox ${ }^{39}$, J. Wood ${ }^{23}$, G.B. Yodh ${ }^{35}$, A. Zepeda ${ }^{4}$, H. Zhou ${ }^{30}$

${ }^{1}$ Barnard College, New York, NY, USA, ${ }^{2}$ Department of Chemistry and Physics, California University of Pennsylvania, California, PA, USA, ${ }^{3}$ Centro de Investigación en Computación, Instituto Politécnico Nacional, Ciudad de México, México, ${ }^{4}$ Physics Department, Centro de Investigación y de Estudios Avanzados del IPN, Ciudad de México, México, ${ }^{5}$ Colorado State University, Physics Dept., Fort Collins, CO, USA, ${ }^{6}$ DCI-UDG, Leon, Gto, México, ${ }^{7}$ Erlangen Centre for Astroparticle Physics, Friedrich Alexander Universität, Erlangen, BY, Germany, ${ }^{8}$ Facultad de Ciencias Físico Matemáticas, Benemérita Universidad Autónoma de Puebla, Puebla, México, ${ }^{9}$ School of Physics and Center for Relativistic Astrophysics, Georgia Institute of Technology, Atlanta, GA, USA, ${ }^{10}$ School of Physics Astronomy and Computational Sciences, George Mason University, Fairfax, VA, USA, ${ }^{11}$ NASA Goddard Space Flight Center, Greenbelt, MD, USA, ${ }^{12}$ Instituto de Astronomía, Universidad Nacional Autónoma de México, Ciudad de México, México, ${ }^{13}$ Instituto de Ciencias Nucleares, Universidad Nacional Autónoma de México, Ciudad de México, México, ${ }^{14}$ Instituto de Física, Universidad Nacional Autónoma de México, Ciudad de México, México, ${ }^{15}$ Institute of Nuclear Physics, Polish Academy of Sciences, Krakow, Poland, ${ }^{16}$ Instituto de Física de São Carlos, Universidade de São Paulo, São Carlos, SP, Brasil, ${ }^{17}$ Instituto de Geofísica, Universidad Nacional Autónoma de México, Ciudad de México, México, ${ }^{18}$ Instituto Nacional de Astrofísica, Óptica y Electrónica, Tonantzintla, Puebla, México, ${ }^{19}$ INFN Padova, Padova, Italy, ${ }^{20}$ Tecnologico de Monterrey, Escuela de Ingeniería y Ciencias, Ave. Eugenio Garza Sada 2501, Monterrey, N.L., 64849, México, ${ }^{21}$ Physics Division, Los Alamos National Laboratory, Los Alamos, NM, USA, ${ }^{22}$ Max-Planck Institute for Nuclear Physics, Heidelberg, Germany, ${ }^{23}$ NASA Marshall Space Flight Center, Astrophysics Office, Huntsville, AL, USA, ${ }^{24}$ Department of Physics and Astronomy, Michigan State University, East Lansing, MI, USA, ${ }^{25}$ Department of Physics, Michigan Technological University, Houghton, MI, USA, ${ }^{26}$ Space Science Center, University of New Hampshire, Durham, NH, USA, ${ }^{27}$ The Ohio State University at Lima, Lima, OH, USA, ${ }^{28}$ Department of Physics, Pennsylvania State University, University Park, PA, USA, ${ }^{29}$ Department of Physics and Astronomy, University of Rochester, Rochester, NY, USA, ${ }^{30}$ Tsung-Dao Lee Institute and School of Physics and Astronomy, Shanghai Jiao Tong University, Shanghai, China, ${ }^{31}$ Sungkyunkwan University, Gyeonggi, Rep. of Korea, ${ }^{32}$ Stanford University, Stanford, CA, USA, ${ }^{33}$ Department of Physics and Astronomy, University of Alabama, Tuscaloosa, AL, USA, ${ }^{34}$ Universidad Autónoma del Estado de Hidalgo, Pachuca, Hgo., México, ${ }^{35}$ Department of Physics and Astronomy, University of California, Irvine, Irvine, CA, USA, ${ }^{36}$ Santa Cruz Institute for Particle Physics, University of California, Santa Cruz, Santa Cruz, CA, USA, ${ }^{37}$ Universidad de Costa Rica, San José, Costa Rica, ${ }^{38}$ Department of Physics and Mathematics, Universidad de Monterrey, San Pedro Garza García, N.L., México, ${ }^{39}$ Department of Physics, University of Maryland, College Park, MD, USA, ${ }^{40}$ Instituto de Física y Matemáticas, Universidad Michoacana de San Nicolás de Hidalgo, Morelia, Michoacán, México, ${ }^{41}$ FCFM-MCTP, Universidad Autónoma de Chiapas, Tuxtla Gutiérrez, Chiapas, México, ${ }^{42}$ Department of Physics and Astronomy, University of New Mexico, Albuquerque, NM, USA, ${ }^{43}$ University of Seoul, Seoul, Rep. of Korea, ${ }^{44}$ Universidad Politécnica de Pachuca, Pachuca, Hgo, México, ${ }^{45}$ Department of Physics, University of Wisconsin-Madison, Madison, WI, USA, ${ }^{46}$ CUCEI, CUCEA, Universidad de Guadalajara, Guadalajara, Jalisco, México, ${ }^{47}$ Universität Würzburg, Institute for Theoretical Physics and Astrophysics, Würzburg, Germany, ${ }^{48}$ Department of Physics and Astronomy, University of Utah, Salt Lake City, UT, USA, ${ }^{49}$ Department of Physics, Faculty of Science, Chulalongkorn University, Pathumwan, Bangkok 10330, Thailand, ${ }^{50}$ National Astronomical Research Institute of Thailand (Public Organization), Don Kaeo, MaeRim, Chiang Mai 50180, Thailand, ${ }^{51}$ Department of Physics, Catholic University of America, Washington, DC, USA, ${ }^{52}$ Center for Research and Exploration in Space Science and Technology, NASA/GSFC, Greenbelt, MD, USA, ${ }^{53}$ Instituto de Física Corpuscular, CSIC, Universitat de València, Paterna, Valencia, Spain 